a new method of preparing the skin for inoculation was brought to my mind because of my sympathy for little children who are frightened and even given pain by the old, scarification plan.

The protection from absorption possessed by the skin is due to the epidermis and chiefly to the horny layer. This being removed, or even scarified, permits absorption. An excoriation or denudation is necessary to local infection in most cases, though follicular inoculation has been credited. Given, then, an impermeable covering, it was but necessary to find an agent which would dissolve or disintegrate the upper layers of the epidermis without pain or fright to the patient. Salicylic acid in glycerin was tried and discarded. A saturated solution of caustic potash in a 20 per cent. solution of cocain was effective, though the final product of the mixture was uncertain. A light application of the caustic potash stick was tried, being neutralieed with acetic acid as soon as burning was felt. ${ }^{1}$ This was effective, but acted rather severely, and left some pigmentation from slight inflammation. Finally, ordinary liquor potassæ was tried and found to meet every indication.

The elder Hebra denuded his old eczema patches with green soap and rubbing, and this softening action of potassa has long been well known. We use liquor potassæ in mounting scales and hairs for microscopic examination, and it rapidly disintegrates them. The pigmentary deposits in the epidermis are easily remuved after the application of liquor potassæ. Officinal liquor potassæ is a 5 per cent. solution, that in common use becomes stronger through evaporation.

The procedure of preparing the surface for vaccination is as follows: The point to be vaccinated is cleansed. A piece of cotton as large as the desired denudation is wet with liquor potassæ and laid on, or a little of the fluid is put on with the bottle stopper. After two or three minutes, or as soon as sliight burning is felt--it usually does not burn at all-the cotton is removed, if it was used, the soap mixture which has formed with the skin secretions is wiped off with a piece of wet cotton, though this is not essential to success, in order to render the next step easier. Then an ink eraser, a tooth-pick of soft wood, a pencil rubber, a piece of gauze (the quickest) or a piece of damp cotton is used to rub away the softened epidermis. The friction is slight, the pain is only a little stinging when the sensory nerve filaments become exposed. We obtain in a few seconds a moist, shining surface, often a clear view of the papillary vessels but no bleeding. The vaccine is now applied and let dry on in the usual way.

The advantages of this method are its practical painlessness and the absence of terrifying instruments, such as the "Neptune's trident," the lancet or sewing needle. Further, bleeding is a bar to successful inoculation. By scarification it is difficult to stop short of bleeding, while with denudation bleeding is almost impossible. A little girl aged 6 years, vaccinated her. self by my method. There is also less danger of an undesired infection from instrument or epidermis when this method is employed.

As to the results: A good lymph will "take"; an unreliable one will not. Inoculation was successful in as many cases vaccinated by denudation as by scar-

Since writing the above the caustic potash stick was tried again, in the absence of a supply of liquor potassa. A minute drop of the delicluescing potash was put on, gently smoothed out and immedi the pain nil and the procedure even more rapid than scarification. ification. Within three days one young man developed twenty-five distinct and characteristic vesicles in an area three-fourths of an inch square. Others "took" in the usual way. Abortive lesions formed in other cases just as after the old method.

One doctor vaccinated seven children in an institution at his first trial of my method. With three he did not wash off the liquor potassæ before denuding; in four he did. Every one of the seven "took." The doctor was fortunate in his lymph. Other physicians have found the method successful. Many cases could not be followed up. Increased experience with the method and the reports of others demonstrate probably a larger percentage of success than is possible with scarification.

Ordinarily there may be one objection to the method of denudation; it is alower, but the time necessary to subdue a refractory child for scarification will often amount to more than two or three minutes. Again, if we draw blood in scarifying, this must all be removed and further flow checked to insure a result; this takes time. For those who wish to save themselves annoyance, or to spare little children and nervous people pain and fright, the method described is preferable. Sympathy and convenience justify its use even if the whole matter is simple and small.

Denudation offers a field for dermic medication which may reduce the necessity for so much use of the hypodermic syringe. I have not experimented here except with cocain and eucain solutions. I obtained their characteristic effects. Something similar to this has been used for the dermic absorption of drugs, viz., the formation of a blister which resulted in the denudation of an absorbent surface. A denudation maintained with moist rubber tissue might spare some ill person frequent, painful thrusts of the hypodermic needle, where such introduction of drugs should be practiced.

Note.--This method was demonstrated to the A tlanta Society of Medicine on the evening of Sept. 21, 1897, a report of which will be published. Most of the experiments with the method were made since September 1 of this year, although slight trial of other agents was had some months ago.

311-312 Fitten Building.

\section{THE DRY METHOD IN INTRA-UTERINE SURGERY.}

Read before the Illinois State Medical Society. BY EDWIN WALKFR, M.D., PH.D.

EVANSVYLIE, IND.

Last year I read before this society a paper on the abuse of water in surgery (AMERICAN MEdical Association Journal, No. 26, 1896). Some of the disadvantages of its excessive use in both septic and aseptic cases was pointed out. It was urged also, that outside of hospitals, where we have to rely on unskilled. help to prepare the water, it is often used from vessels. improperly cared for, and is often the source of infec. tion. On the other hand, there is no longer a doubt. that ideal asepsis may be attained by the dry method. The action of the water, though it be pure and sterile, exercises an unfavorable influence on healthy tissues, while antiseptic solutions often injure them and mark. edly reduce their ability to resist infection. It is practically settled, that septic wounds can not be rendered aseptic by any washing, or the use of antiseptics, no matter how strong they are used; all we can do is to render the conditions such as to give the tissues a chance to exclude the germs. 
It is not intended to inveigh against water used to cleanse the parts before operation, for it is here indispensible, but after the operation is begun it should be dispensed with; nor is it denied that in some septic cases, as ulcers or abscess cavities. it may be mechanically impossible to remove sloughs or other detritus without water. If efficient drainge is established, such cases will not he numerous.

In this paper I will discuss the dry method in intrauterine work. I believe there has been almost a universal tendency in this direction; personally I have gradually used less irrigation, and now have almost entirely given it up, and my results have since heen much more satisfactory.

The most frequent occasion for invasion of the uterine cavity, is to remove the products of conception, and curettage for indometritis, and in these cases I have most extensively employed the dry method. It has been almost the universal custom in these cases to irrigate with plain water, or some antiseptic solution; this is directed by all the standard works on gynecology. Irrigation is superfluous. After a thorough curettage for endometritis or the removal of the placenta, we have a fresh wound which is to be cleaned, the blood wiped off just as from an incision. This can be more efficiently done by wiping with sterile gauze than in any other way. Irrigation is often positively harmful. Who has not seen localized peritonitis after irrigation of the uterus, from passage of fluid through the fallopian tube? Besides, the water or antiseptic does in some degree impair the exposed tissues, and is often the carrier of infection. It is a rule that the simplest method, the one which requires the employment of the fewest things and the least handling, is the one which will most frequently attain asepsis. Water or fluids have to be poured from one vessel to another, and in many ways exposed to contamination.

By the dry method these cases are very simple. In curettage for endometritis, the patient is put in Simon's position and the vagina scrubbed with liquid soap. This is washed out with creolin. and followed by plain water. It is then thoroughly dried with sterile cotton, dilated and thoroughly curetted as usual. When this is done the cavity is thoroughly wiped out with sterilized gauze, the wiping being repeated several times, until the gauze comes out but slightly tinged with bloöd. Then it is wiped out with iodoform gauze, and packed with the same. I have found it a great help to have the gauze, both plain and iodoform, cut in strips and packed in a glass tube (a large test tube). It is sterilized in these tubes, and drawn directly from them as placed in the uterus, without touching anything but the dressing forceps. If the case is one of abortion, and part or all of the ovum is retained, the same method is followed, except that Martins' curettage forceps are used to facilitate the emptying of the organ. These are dull, smaller and less clumsy than the ordinary placental forceps, and are much more convenient and efficient. The wiping and packing is the same, except that it has to be more frequently done, the hemorrhage is greater, and there are more shreds of tissue to be wiped out. I do not suppose that by this process, we really remove all the membranes; I doubt if we ever do, but this is not necessary, for in aseptic treatment healing will be complete. If the os is patulous and the abortion is as late as the fourth or fifth month, the fingers may be introduced and will greatly aid in the removal of the pla- centa. I have, however, found the forceps a great aid in these advanced cases, and have never done any damage with them. I have in two instances emptied the uterus for vomiting in pregnancy. These cases had both advanced to about the eighth week. The romiting was so severe and constant, that after consultation it was decided that farther delay would greatly endanger life. Theos was dilated as usual, theovum was seized with forceps and stripped out, the membranes being extracted in pieces. The cavity was then curetted, wiped out and packed. In neither case did the temperature rise over 99.6, nor the pulse above normal. In both cases the nausea ceased at once, and neither was sick from the anesthetic. It is interesting to note here, that one patient became pregnant a few months later, and was almost entirely free from nausea during her pregnancy. I have a number of times emptied the uterus in a similár manner, before fetus was expelled, after finding that abortion was inevitable. The ad. advantage is, that the patient is spared much suffering and hemorrhage, and the danger is very much less than by waiting for nature to do the work.

I can not leave this part of the subject without a not of warning. It is understood that this method is free from danger only in the hands of those who have sufficient skill to do it properly, and have learned by careful study and experience to do aseptic work. Unless these manipulations are done with care and under strictest asepsis, many accidents will occur. But these are chargeable to the individual, not to the method.

When the contents of the uterus has become septic the method should be the same; dilate, remove contents, wipe out and pack. I feel that my experience in abortions justifies me in commending it as the best and safest. In puerperal cases, my experience has not been sufficient to speak authoratively, but I do not believe it will prove an exception. In one case of missed abortion, my colleague, Dr. Owen, removed a septic ovum of three and a half or four months, in this manner. The patient became pregnant, the fetus for some reason died between the third and fourth month. Later she had fever. This continued three months. When she came under the doctor's care she was much reduced by long pyrexia; examination revealed the condition. She made a rapid recovery. This case was an excellent test; notwithstanding the ovum was torn out, and not a drop of water was used, the result was perfect. In ordinary cases where the fetus has been expelled and the placental membrane remains, and fever has set in, the dry method leaves nothing to be desired.

The only cases in which there is any doubt of its efficiency, are the puerperal or later abortions, in which the uterus is markedly affected and a large amount of pus or septic material is found. These are the cases in which frequent or constant irrigation has been recommended, and in many cases is doubtless successful. In private practice this is a very difficult method to carry out. It is not safe to trust it to a nurse, and it requires almost constant presence of the physician, and besides, in many cases it fails. I believe the manual disturbance in these cases, is, in many instances, bad. The barrier against infection, which nature throws out around any area to shut off systemic infeotion, is often broken down by such frequent interference. It may be urged that the packing would be, but this latter need not be so often done, and if carefully executed would not do as much violence as the 
intra-uterine douche. If this could be replaced by packing which would not require removal oftener than once or rarely twice in twenty-four hours, it would be a great gain. The gauze takes up much of the discharge and keeps the os open, thus securing drainage. I have recently used the packing in two cases, one of which recovered, but in neither case was it a fair test, as it was not begun early, nor was it used alone.

\section{A CASE ILlUSTRATING THE VALUE OF SYMPHYSIOTOMY WHEN A TUMOR OBSTRUCTS THE PARTURIENT CANAL.}

Read before the Denver and Arapahoe County Medical Society, February, 1897

BY H. W. ROVER, M.D.

IECTURER ON THERA PEUTICS, DENVER MEDICAL COLLEGE. AND T. MITCHELL BURNS, M.D.

PROFESSOR OF OBSTETRICS, GROSS MEDICAL COLLEGE, DENYER, COLO.

PART I.-BY DR. ROVER. INTRODUCTION.

Based on two cases of symphysiotomy which occurred in my private practice (one operated on by $D r$. Gustav Zinke of Cincinnati, and the other by Dr. T. M. Burns of Denver), and both of them successful to mother and child, I desire to give expression and emphasis to the benefits derived from said operation.

The operation, though a feasible one, does not suggest itself to every medical mind interested in any particular case. The patient was seen repeatedly by three physicians during the latter part of her pregnancy and no unanimity of opinion could be reached; not even after she was taken in labor did the various counsel agree. One favored a Porro, the second a craniotomy, I pleaded for a symphysiotomy. Why this discrepancy of opinion? Simply because the respective indications, benefits and limitations for either of these operations are not definitely fixed.

It may be said that under favorable conditions and at the hands of skilled operators, the death-rate has been almost nil. Pinard lost but one mother in his first twenty operations, and Sweifil one in forty-three cases. The fetal mortality, including those that die shortly after birth, runs higher; according to Neugebauer as high as 20.5 per cent.

Cæsarian section, although more saving to child life, is more dangerous to the mother. Induced premature labor under approved modern methods with its low maternal death-rate, is more hazardous to the fetus than is a symphysiotomy.

Where a symphysiotomy is possible and the child in utero is viable, we should never think of performing a craniotomy. In this connection $I$ recall the teachings of an old professor (Dr. Tate of Cincinnati) who declared that an unborn child, whose life is being held at stake, may prove to be an unborn Milton or Shakespeare; hence the conclusion that if other safe measures can be instituted the fetal life is not to be sacrificed.

The woman's powers of locomotion are not necessarily crippled by slight looseness of the joint, and as a rule, under a rigid asepsis and with complete immobilization of the pelvis during convalescence, the restoration of the symphysis in women not previously infected is complete. In both of my cases the union was perfect, in the first, great precautions were taken to secure the immobilization of the pelvis by the use of both adhesive plaster and a jacket of plaster-of. Paris; whereas, in the second case only a strong linen many-tailed bandage held the parts together.

The operation is best adapted to the symmetrical rachitic pelvis, and should not be undertaken when the true conjugate is less than two and five-eighth inches. As much as two to three inclies of separation may be secured without risk of injury to the sacroiliac symphysis. Version should not be resorted to when the head can be made to engage.

Case report.-Mrs. K., German, aged 35 years, married seven years, childhood history negative, no rachitic tendencies.

Her labors: 1st, at nine months vertex, terminated by craniotomy after nine hours of labor; $2 \mathrm{~d}$, at nine mon ths vertex, terminated by podalic version after five hours of labor; $3 \mathrm{~d}$, at eight months breech, terminated by inducing labor with the catheter. Catheter was in place six hours before labor set in, the labor lasting six hours ; 4 th, occurred under my supervision Aug. 10,1895 . When consulted beforehand I advised a symphysiotomy, but the patient had been instructed by the physician in charge of her previous confinements that a premature labor induced at the seventh month, and the mother during her pregnancy resorting to a vegetarian diet (particularly fruit), would offer the best hope for a successful issue on the part of child and mother. As in the third pregnancy, the catheter was introduced but remained in place for two days before labor set in. The labor lasted six hours and the presentation was a breech. Unfortunately prolapsus of the cord occurred early in the labor, and as its result a dead baby was born; 5 th, this was terminated by the symphysiotomy reported in this article and resulted in saving both child and mother.

The puerperal state in each instance was normal, and with the exception of the last she was up on the eighth day. Her various pregnancies were all normal.

History of tumor.-Patient never felt any tumor or symptoms from it. At the first labor (which took place in Germany) the attending physician diagnosed a contracted pelvis due to the sacral prominence projecting abnormally forward; at the second labor she had the same physician and consultant. There was nothing new with reference to the obstruction. At the third labor she had the same physician. I advised symphysiotomy during the fourth, but by request induced labor at seven months. All I could make out was a dystocia due to abnormal size of the promontory. In all of the first four pregnancies the child was still-borm but living until artificial aid was used. Her last labor, the fifth, began July 27, 1896, at 7 A.M., and the waters broke at 2 P.M. I saw her at 3 P.M., and a little later the other two consultants were at the bedside. No unanimity of opinion, as before stated, could be reached, each of the three physicians contending for his choice of operation. The family decided against the Porro; the vitality of the child decided against a craniotomy, and hence about 5 P.M. Dr. Burns was called in to perform the symphysiotomy, he advocating this measure. Her previous pregnan. cies, with their respective terminations, warranted the step taken, and today there is happiness in a Denver home because of such a proceeding.

PART II.-BY DR. BURNS.

THE OPERATION, SUBSEQUENT TREATMENT AND RESULTS,

By abdominal palpation, the presentation was found to be a vertex, and the position a left occipitoposterior. The forehead was prominent on the right side above the brim, an indication of some obstruction to the engagement of the presenting part.

By pelvimet $y$, the distance between the anterior superior spines was ten and one-half inches, the crests eleven inches and the external conjugate seven inches. These measurements indicated that the dimensions of 